GU J Sci, Part C, 5(4): 201-213 (2017)

Gazi Üniversitesi
Fen Bilimleri Dergisi
PART C: TASARIM VE TEKNOLOJI
dergipark.gov.tr/http-gujsc-gazi-edu-tr

\title{
Kaiser ve Von-Hann Pencereleri Tabanlı Yeni bir Pencere Fonksiyonu ve Onun Sayısal Süzgeç Tasarımındaki Performans Analizi
}

\author{
Kemal AVCi $\dot{1}^{1, *}$ \\ ${ }^{l}$ Abant İzzet Baysal Üniversitesi, Mühendislik ve Mimarllk Fakültesi, Elektrik ve Elektronik Mühendisliği Bölümü, 14300, BOLU
}

\begin{abstract}
$\ddot{O} \mathbf{z}$
Makale Bilgisi

Bașvuru: $23 / 08 / 2017$

Düzeltme: $19 / 11 / 2017$

Kabul: 20/11/2017

Anahtar Kelimeler

Sayısal süzgeçler Pencere fonksiyonlart Kaiser-Hann penceresi Kaiser penceresi

Birçok sinyal işleme uygulamasında kullanılan sayısal süzgeçler, yinelemeli veya yinelemesiz olarak sınıflandırılırlar. Daima kararlı ve tam doğrusal faz karakteristiği sağlayabildiği için yinelemesiz sayısal süzgeçler literatürde önemli bir yer tutmaktadır. Bu makalede, yinelemesiz sayısal süzgeçlerin tasarımı için yeni bir hibrit pencere fonksiyonu önerilmektedir. Bu yeni pencere, literatürde Kaiser ve Von-Hann olarak bilinen iki farkl1 pencere fonksiyonun birleştirilmesiyle elde edilmiştir. İlk olarak, önerilen pencere fonksiyonun sahip olduğu iki bağımsız parametrenin sayısal süzgeç karakteristiği üzerindeki etkileri, minimum durdurmabandı zayıflatması ve geçiş bant-genişliği cinsinden analiz edilmiştir. Daha sonra, önerilen pencere fonksiyonunun sayısal süzgeç tasarımındaki performansının literatürde iyi bilinen diğer iki parametreli pencere fonksiyonlarıyla kıyaslamak için farklı süzgeç uzunluklarında karşılaştırma örnekleri verilmiştir. Benzeşim sonuçları, önerilen pencere fonksiyonun KaiserHamming, Cosh-Hamming, Exponential-Hamming, Saramaki, Kaiser, Dolph-Chebychev, Cosh, Exponential, Gaussian, Hann-Poisson ve Poisson pencere fonksiyonlarına kıyasla daha iyi süzgeç tasarımı sağlayabildiğini göstermektedir.
\end{abstract} Von-Hann penceresi

Keywords

Digital filters Window functions Kaiser-Hann window Kaiser window Von-Hann window

\section{A New Window Function Based on Kaiser and Von-Hann Windows and its Performance Analysis for Digital Filter Design}

\begin{abstract}
Digital filters, which are used in many signal processing applications, can be classified as recursive or nonrecursive. Since nonrecursive digital filters can provide inherent stability and exact linear phase characteristic, they have an important place in the literature. In this paper, a new hybrid function is proposed for the design of nonrecursive digital filters. This new window was obtained by combining two different window functions known as Kaiser and Von-Hann in the literature. First of all, the effects of two independent parameters of the proposed window function on the digital filter characteristic are analyzed in terms of minimum stopband attenuation and transition bandwidth. Later, comparative examples for different filter lengths are given to compare the performance of the proposed window function in the design of digital filter with the well-known other two parameter window functions. Simulation results demonstrate that the proposed window function can provide a better filter design than Kaiser-Hamming, CoshHamming, Exponential-Hamming, Saramaki, Kaiser, Dolph-Chebychev, Cosh, Exponential, Gaussian, Hann-Poisson and Poisson window functions.
\end{abstract}

\section{GIRISŞ (INTRODUCTION)}

Süzgeçler, bir işaret içerisindeki istenmeyen bileşenlerin zayıflatılmasını sağladıklarından elektronik mühendisliğinin birçok uygulamasında kullanılan önemli yapılardır. Gerçekleştirilmelerinde kullanılan fiziksel yapılara göre süzgeçler, analog veya sayısal olarak sinıflandırılırlar. Sayısal süzgeçlerin sayısal teknolojinin avantajlarını taşımalarından dolayı, işaret işleme uygulamalarında sıklıkla kullanılırlar. Sayısal süzgeçler, çıkış işaretinin hesaplanmasında çıkış işaretinin önceki değerlerinin kullanılıp kullanılmamasına göre yinelemeli veya yinelemesiz olarak sınıflandırılmaktadırlar [1]. Yinelemesiz süzgeçlerin daima kararlı yapıda olmaları ve kolayca tam doğrusal fazlı olarak tasarlanabilmeleri nedeniyle ve ayrıca bu özelliklerin 
birçok işaret işleme uygulamasında istenilen özellikler olmasından dolayı, yinelemesiz süzgeçlerin tasarımı literatürde önemli bir yer tutmaktadır.

Pencereleme yöntemi, hesaplama avantajından dolayı yinelemesiz sayısal süzgeçlerin tasarımında kullanılan önemli yöntemlerden biridir. Pencereleme yönteminde pencere fonksiyonları (veya kısaca pencere) kullanılmakta olup, pencereleme işlemi ile süzgeç genliğinde oluşan istenmeyen Gibbs salınımlarının azaltılması amaçlanmaktadır [1]. Pencereleme tabanlı sayısal süzgeç tasarımları optimum sonuçlar vermediğinden, daha iyi süzgeçler elde etmek için literatürde birçok pencere önerilmiştir [2-16]. Pencereler, fonksiyonlarında sahip oldukları bağımsız parametre sayısına göre sabit veya ayarlanabilir pencere olarak sinıflandırılmaktadırlar [6]. Sabit pencereler sinıfinda olan Rectangular, Von-Hann, Hamming ve Blackman pencereleri [1], fonksiyonlarında sadece bir bağımsız parametreye sahip olup bu parametre ise pencere uzunluğudur. Dolayısıyla, sabit pencereler sabit bir süzgeç uzunluğu için sadece bir süzgeç karakteristiği sunmaktadırlar. Sabit pencerelerin sahip olduğu elastikiyet problemini gidermek için, en az iki bağımsız değişkene sahip olan çeşitli ayarlanabilir pencereler önerilmiştir. Ayarlanabilir pencereler içerisinde sayısal süzgeç tasarımında en çok bilinen ve kullanılan pencere, Kaiser penceresidir [4].

$\mathrm{Bu}$ çalışmada, literatürde çok iyi bilinen Kaiser penceresine göre daha iyi süzgeç karakteristiği sağlayabilecek yeni bir pencere önerilmiştir. $\mathrm{Bu}$ pencere, Kaiser ve Von-Hann pencerelerinin birleştirilmesinden elde edilmiş ve bu çalışmada kısaca Kaiser-Hann penceresi olarak isimlendirilmiştir. İkinci bölümde pencere fonksiyonu tanımı ile birlikte önerilen pencerenin nasıl tanımlandığı verilmiştir. Üçüncü bölümde ise pencere kullanarak sayısal süzgeç tasarımının nasıl yapıldığı anlatılmıştır. Dördüncü bölümde ise önerilen pencerenin sayısal süzgeç tasarımındaki performans analizi kıyaslamalı örneklerle birlikte verilmiştir. Çalışma ile ilgili önemli sonuçlar son bölümde özetlenerek sunulmuştur.

\section{2. ÖNERILEN PENCERE FONKSIYONU (THE PROPOSED WINDOW FUNCTION)}

$\mathrm{Bu}$ bölümde bir pencere fonksiyonun tanımı ile süzgeç tasarımı için önerilen pencere fonksiyonu verilmiştir.

\subsection{Pencere Fonksiyonun Tanımı (Definition of Window Function)}

Bir pencere fonksiyonu, belirli bir zaman aralığında sıfırdan farklı ve o zaman aralığı dışındaki aralık için sıfir değerine sahip zaman alanlı bir fonksiyondur [1]. Ayrık zamanlı bir pencere fonksiyonu, w(n) olarak gösterilmekte olup örnek zaman uzunluğunda tanımlanmaktadır. Ayrık zamanlı simetrik bir pencerenin normalize edilmiş genlik karakteristiği, genelde Şekil 1'deki gibidir.

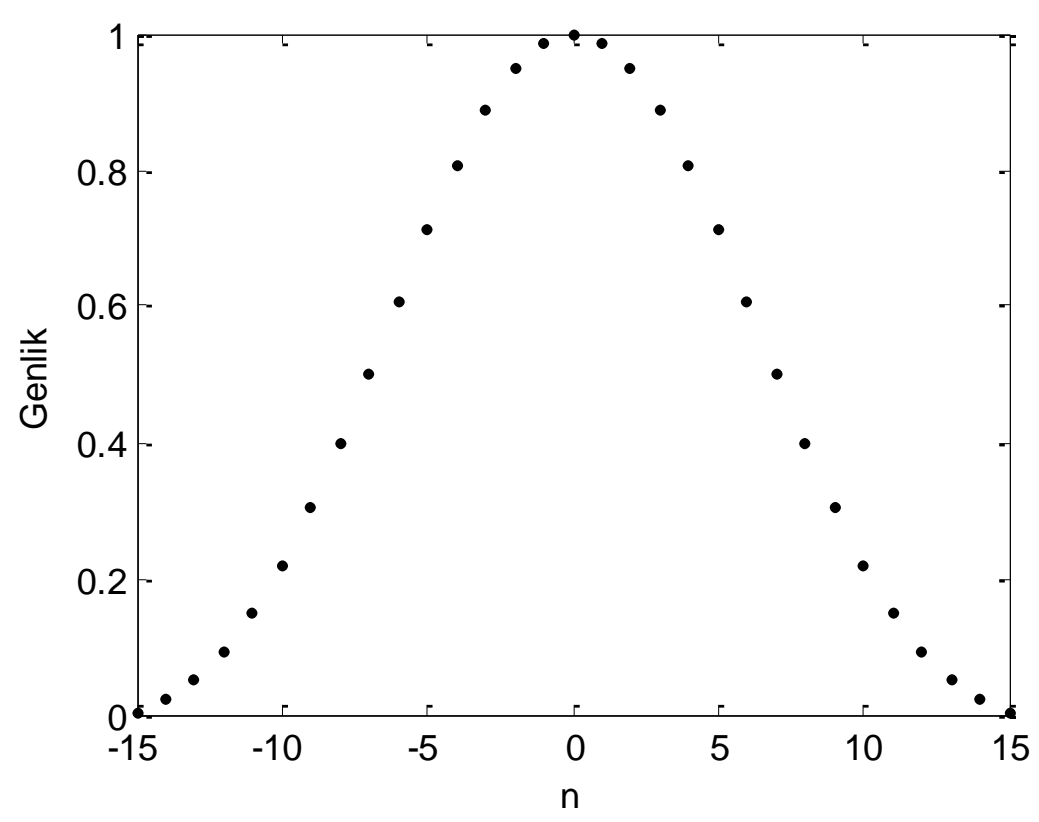

Şekil 1. Ayrık zamanlı bir pencerenin genlik karakteristiği $(N=31)$ 


\subsection{Kaiser Penceresi (Kaiser Window)}

Ayrık zamanlı Kaiser penceresi Denklem (1)'te belirtildiği gibi tanımlanmakta olup, N pencere uzunluğunu ve $\alpha$ ayarlanabilir bağımsız parametreyi göstermektedir [4].

$$
w(n)=\frac{I_{0}\left(\alpha \sqrt{1-\left(\frac{2 n}{N-1}\right)^{2}}\right)}{I_{0}(\alpha)} \quad|\mathrm{n}| \leq(\mathrm{N}-1) / 2 \text { için }
$$

$\mathrm{Bu}$ penceredeki $I_{0}(x)$ ise Denklem (2) ile ifade edilen sıfır dereceli birinci tür geliştirilmiş Bessel fonksiyonunu göstermektedir.

$$
I_{0}(x)=1+\sum_{k=1}^{\infty}\left[\frac{1}{k !}\left(\frac{x}{2}\right)^{k}\right]^{2}
$$

\subsection{Von-Hann Penceresi (Von-Hann Window)}

Von-Hann penceresi, bağımsız değişken olarak sadece pencere uzunluğuna sahip olan bir sabit pencere olup Denklem (3) ile tanımlanmaktadır [1].

$$
w(n)=0,5+0,5 \cos \frac{2 \pi n}{N-1} \quad|\mathrm{n}| \leq(\mathrm{N}-1) / 2 \text { için }
$$

\section{4. Önerilen Pencere: Kaiser-Hann Penceresi (Proposed Window: Kaiser-Hann Window)}

Bu çalışmada önerilen pencere, literatürde tanımlanmış Kaiser ve Von-Hann pencerelerinin Denkem (4)'te verilen hibrit birleşimi olarak tanımlanmıştır.

$$
w(n)=\frac{I_{0}\left(\alpha \sqrt{1-\left(\frac{2 n}{N-1}\right)^{2}}\right)}{2 I_{0}(\alpha)}+0,25+0,25 \cos \frac{2 \pi n}{N-1} \quad|\mathrm{n}| \leq(\mathrm{N}-1) / 2 \text { için }
$$

Önerilen pencere iki bağımsız değişkene sahip olup, bunlar N pencere uzunluğu ve $\alpha$ ayarlanabilir biçim parametreleridir. Bu iki parametrenin süzgeç tasarımı üzerindeki etkileri dördüncü bölümde verilecektir.

\section{PENCERELEME ILE YINELEMESIZ SAYISAL SÜZGEÇ TASARIMI (NONRECURSIVE DIGITAL FILTER DESIGN BY WINDOWING)}

\subsection{Süzgeç Spektral Parametreleri (Filter Spectral Parameters)}

Alçak geçiren bir sayısal süzgeç karakteristiği üzerinden süzgeç spektral parametreleri Şekil 2'de verilmiştir. Şekilde verilen süzgeç spektral parametreleri; geçirme-bandı frekansı $\left(\omega_{\mathrm{p}}\right)$, durdurma-bandı frekansı $\left(\omega_{\mathrm{st}}\right)$, örnekleme frekans1 $\left(\omega_{\mathrm{s}}\right)$, maksimum geçirme-bandı zayıflatması $\left(\mathrm{A}_{\mathrm{p}}\right)$ ve minimum durdurmabandı zayıflatması $\left(A_{s}\right)$ olup bunlar dışında $(5)$ ve (6) eşitlikleriyle tanımlanan kesme frekansı $\left(\omega_{c}\right)$ ve geçiş bant-genişliği $(\Delta \omega)$ parametreleri de bulunmaktadır.

$$
\begin{aligned}
& \omega_{c}=\left(\omega_{s t}+\omega_{p}\right) / 2 \\
& \Delta \omega=\omega_{s t}-\omega_{p}
\end{aligned}
$$




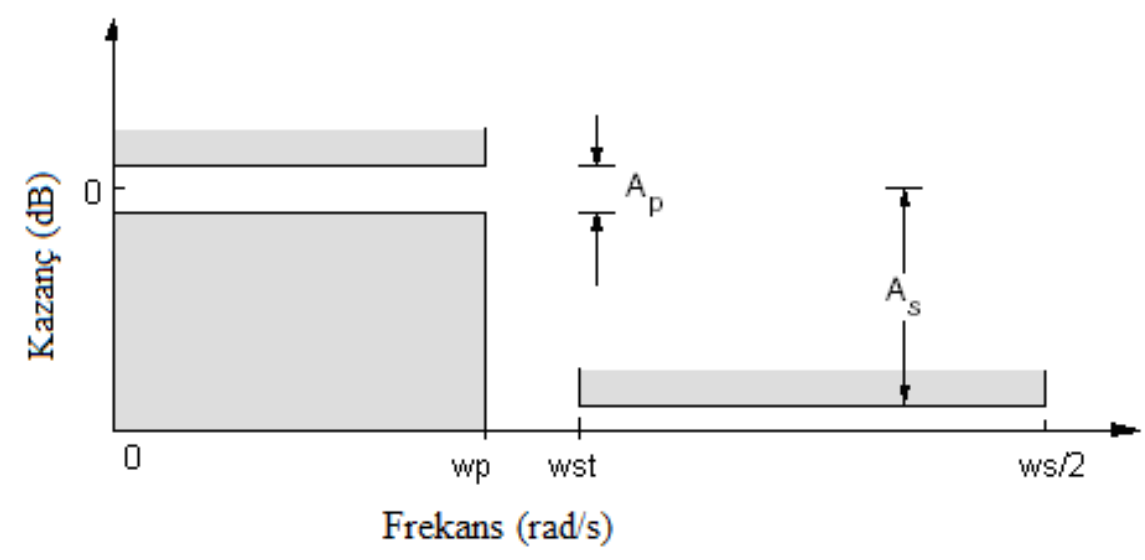

Şekil 2. Alçak geçiren bir süzgecin genlik spektrumu ve süzgeç spektral parametreleri

\subsection{Pencereleme Tabanlı Yinelemesiz Sayısal Süzgeç Tasarımı (Nonrecursive Digital Filter Design Based on Windowing)}

Yinelemesiz sayısal süzgeç tasarımında bir pencere kullanılmasının iki amacı vardır. İdeal süzgecin sonsuz uzunluklu dürtü tepkisini keserek onu sonlu hale getirmek ve istenmeyen Gibss salınımlarını azaltmaktır. Bir $w(n)$ penceresi ve gerçekleştirilemeyen ideal süzgeç kullanarak gerçekleştirilebilir bir süzgecin dürtü tepkisi, Denklem (7) kullanılarak bulunabilir [1].

$$
\boldsymbol{h}(\boldsymbol{n})=w(n) h_{i d}(n)
$$

Burada, $h_{i d}(n)$ ideal süzgecin sonsuz uzunluklu dürtü tepkisi olup, alçak geçiren bir süzgeç için bu fonksiyon Denklem (8)'de verilmiştir [1].

$$
h_{i d}(n)=\left\{\begin{array}{ccc}
\frac{w_{c}}{\pi} & n=0 & i c ̧ i n \\
\frac{s_{i n w_{c}} n}{n \pi} & n \neq 0 & i c ̧ i n
\end{array}\right.
$$

Pencere kullanarak tasarlanan süzgeçlerin, geçirme-bandı ve durdurma-bandı bölgelerinde oluşan istenmeyen salınımlar birbirine eşit olduğundan, benzeşim sonuçları için sadece bir zayıflatma parametresini (genelde $\mathrm{A}_{\mathrm{s}}$ ) hesaplamak yeterlidir.

\section{BENZEŞIM SONUÇLARI (SIMULATION RESULTS)}

Bu bölümde öncelikle zaman bölgesinde önerilen pencerenin zaman karakteristiği incelenmiş ve sonrasında frekans bölgesinde önerilen pencerenin yinelemesiz sayısal süzgeç tasarımındaki performansı karşılaştırmalı örnekler verilerek gözlemlenmiştir.

\subsection{Kaiser-Hann Penceresi Zaman Karakteristiği (Time Characteristic of Kaiser-Hann Window)}

Şekil 3 'te $\mathrm{N}=51$ pencere uzunluğunda önerilen pencerenin farklı $\alpha$ değerlerinde çizilmiş normalize zaman karakteristiği görülmektedir. Her pencerenin 51 adet örnekten oluştuğu ve $\alpha$ değerinin artmasıyla pencere uç kısımlarının sıfıra yaklaştığı gözlemlenmektedir.

Şekil 4'te ise $\alpha=2$ için önerilen pencerenin farklı pencere uzunluklarında çizilmiş normalize zaman karakteristiği görülmektedir. Her pencerenin pencere uzunluğu kadar sıfırdan farklı örnekten oluştuğu ve pencere uzunluğunun artmasıyla pencere uç kısımlarındaki değerlerin değişmediği gözlemlenmektedir. 


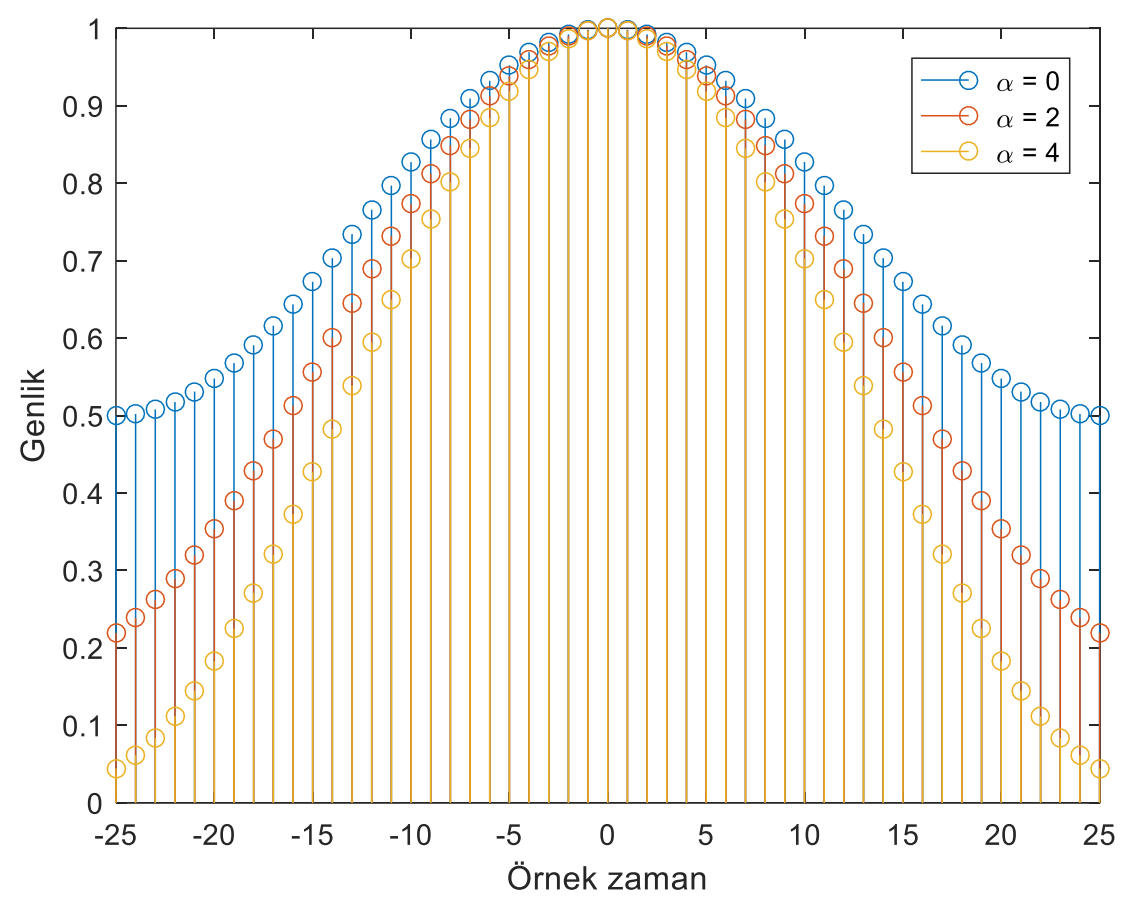

Şekil 3. $N=51$ için farklı $\alpha$ değerlerinde çizilmiş önerilen pencerenin genlik karakteristiği

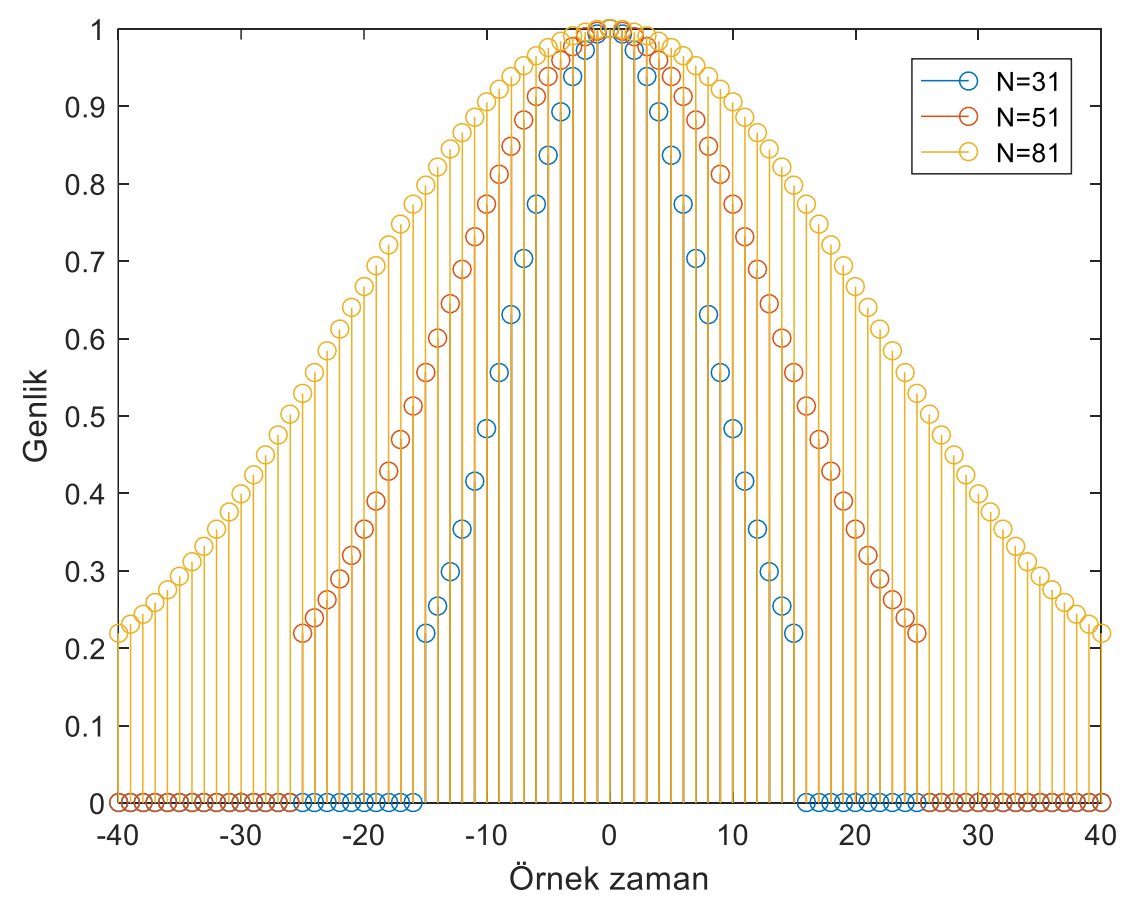

Şekil 4. $\alpha=2$ için farkl pencere uzunluklarında çizilmiş önerilen pencerenin genlik karakteristiği

\subsection{Kaiser-Hann Penceresi Tabanlı Yinelemesiz Süzgeç Tasarımı Benzeşim Sonuçları (Simulation Results of Nonrecursive Filter Design Based on Kaiser-Hann Window)}

Bu kısımda önerilen pencereye ait bağımsız parametrelerin ( $\mathrm{N}$ ve $\alpha$ ) sayısal süzgeç spektrumu üzerindeki etkisi gözlemlenecek ve sonra bu parametrelerin süzgeç spektral parametreleriyle $\left(\mathrm{A}_{\mathrm{s}}\right.$ ve $\left.\Delta \omega\right)$ aralarındaki ilişki bulunacaktır.

Önerilen pencere kullanarak 51 süzgeç uzunluğu ve farklı $\alpha$ değerleri $(\alpha=0,2$ ve 4$)$ için $\omega_{c}=0,4 \pi \mathrm{rad} / \mathrm{s}$ kesim frekansına sahip olarak tasarlanmış alçak geçiren süzgeçlere ait genlik spektrumları Şekil 5'te verilmiştir. Şekil 6' da ise önerilen pencere kullanarak $\alpha=2$ ve farklı süzgeç uzunluklarında $(N=31$, 51 ve 81) için tasarlanmış alçak geçiren süzgeçlere ait genlik spektrumları verilmiştir. Bu iki şekilden elde edilen 
bilgilerle birlikte farklı N ve $\alpha$ değerleri için bulunan süzgeç spektral değerleri Tablo 1'de verilmiştir. Tablo 1 'de, süzgeçlerin geçiş bant-genişliği $(\Delta \omega)$ ve minimum durdurma-bandı zayıflatması $\left(A_{s}\right)$ değerleri, sirasıyla $\mathrm{rad} / \mathrm{s}$ ve $\mathrm{dB}$ cinsinden verilmiştir. Bu tabloda görüldüğü üzere sabit bir süzgeç uzunluğu için $\alpha$ biçim parametresi arttı̆̆ında süzgeç geçiş bant-genişliklerinin genişlemiş (yani ideal durumdan uzaklaşmış) ve minimum durdurma-bandı zayıflatmalarının ise artmış (yani ideal duruma yakınlaşmıştır) olduğu gözlemlenmiştir. Bunun yanı sıra aynı tabloda, sabit bir $\alpha$ değerinde süzgeç uzunluğu arttı̆̆ında, süzgeç geçiş bant-genişliklerinin daralmış (yani ideal duruma yakınlaşmış) ve minimum durdurma-bandı zayıflatmalarının ise çok küçük bir oranda değiştiği gözlemlenmiştir.

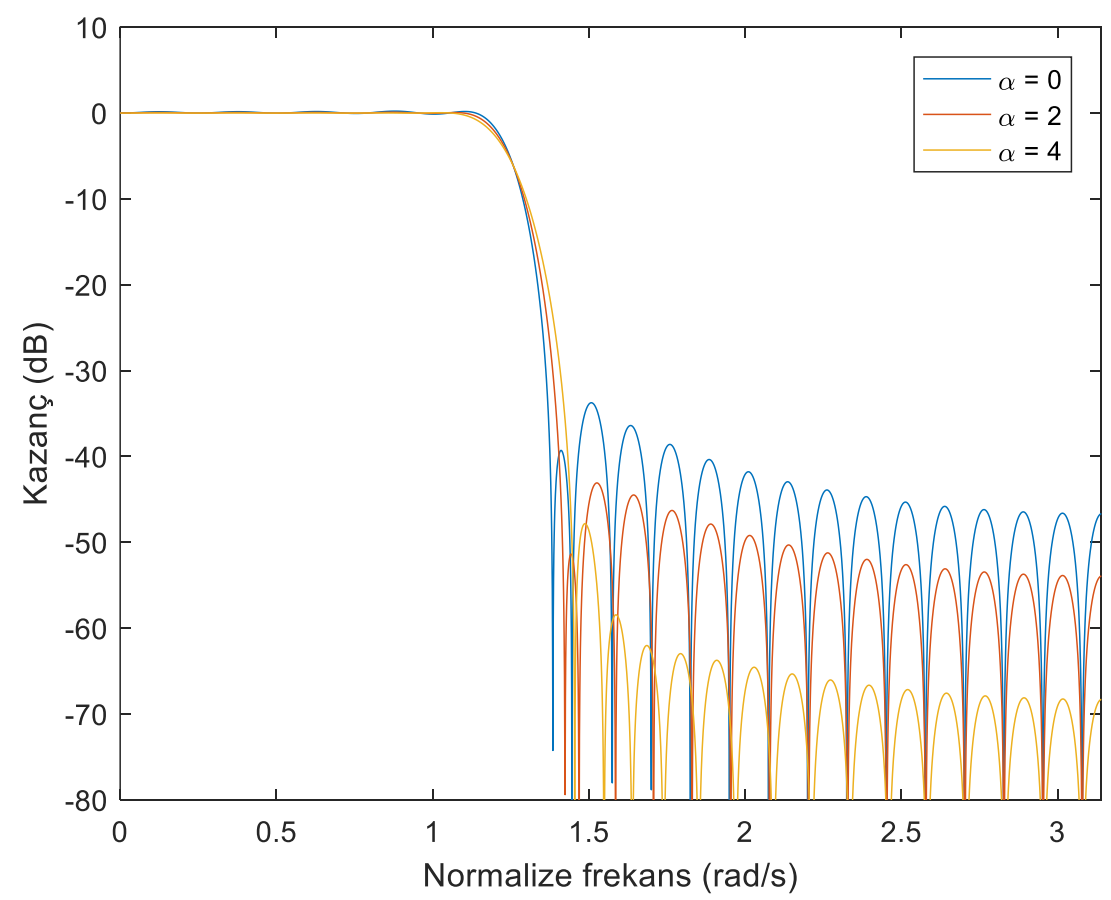

Şekil 5. $N=51$ ile $\alpha=0,2$ ve 4 için önerilen pencereyle tasarlanmıs süzgeçlerin genlik spektrumları

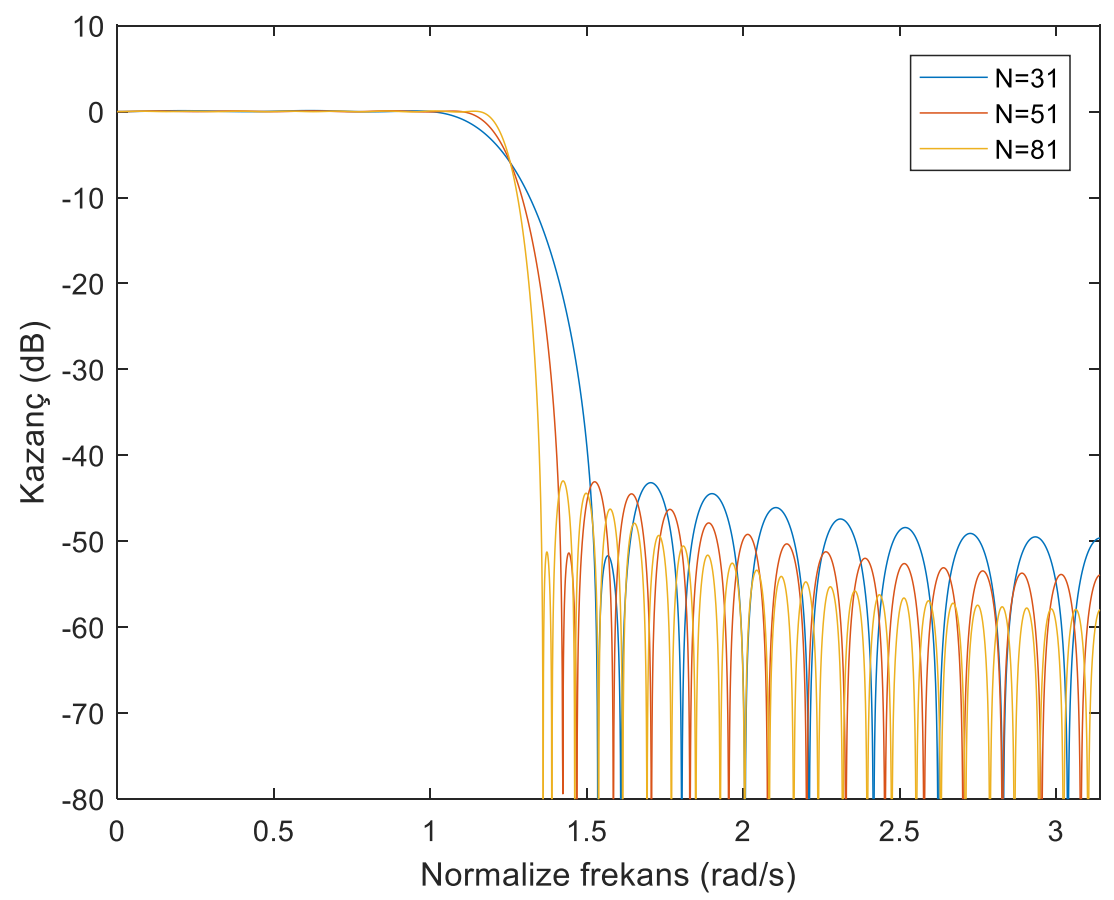

Şekil 6. $\alpha=2$ ve $N=31,51$ ve 81 için önerilen pencereyle tasarlanmış süzgeçlerin genlik spektrumları 
Tablo 1. Farklı $N$ ve a değerleri için önerilen pencereyle tasarlanmış süzgeçlere ait süzgeç spektral değerleri

\begin{tabular}{|c|c|c|c|c|c|c|}
\hline \multirow{2}{*}{$\boldsymbol{\alpha}$} & \multicolumn{2}{|l|}{$\mathbf{N}=\mathbf{3 1}$} & \multicolumn{2}{|c|}{$\mathbf{N}=\mathbf{5 1}$} & $\mathbf{N}=\mathbf{8 1}$ & \\
\cline { 2 - 7 } & $\boldsymbol{\Delta \mathbf { w }}$ & $\mathbf{A}_{\mathbf{s}}$ & $\boldsymbol{\Delta \mathbf { w }}$ & $\mathbf{A}_{\mathbf{s}}$ & $\boldsymbol{\Delta \mathbf { w }}$ & $\mathbf{A}_{\mathbf{s}}$ \\
\hline 0 & 0,368 & 33,77 & 0,221 & 33,74 & 0,139 & 33,68 \\
\hline 2 & 0,502 & 43,18 & 0,302 & 43,08 & 0,189 & 42,98 \\
\hline 4 & 0,638 & 47,86 & 0,382 & 47,83 & 0,239 & 47,84 \\
\hline
\end{tabular}

Önerilen pencere ile tasarlanmış süzgeçlerde, önerilen pencere parametreleri ile süzgeç parametreleri arasında incelenecek ilk ilişki $\alpha$ ve $A_{s}$ arasındaki ilişkidir. Bunun için farklı süzgeç uzunlukları için birçok sayısal süzgeç örneğinden elde edilmiş $\alpha$ ve $\mathrm{A}_{\mathrm{s}}$ arasındaki ilişki Şekil 7'de verilmiştir. Bu şekilde görüleceği üzere yaklaşık olarak $\alpha>3$ için minimum durdurma-bandı zayıflatmasının artık artmadığı (yani daha iyi bir değere gitmediği) görülmektedir. Dolayısıyla, $\mathrm{A}_{\mathrm{s}} \leq 50 \mathrm{~dB}$ aralığında iyi yinelemesiz süzgeç tasarımı için önerilen pencerenin $\alpha \leq 3$ değerleri için kullanılması gerektiği sonucuna varılmıştır.

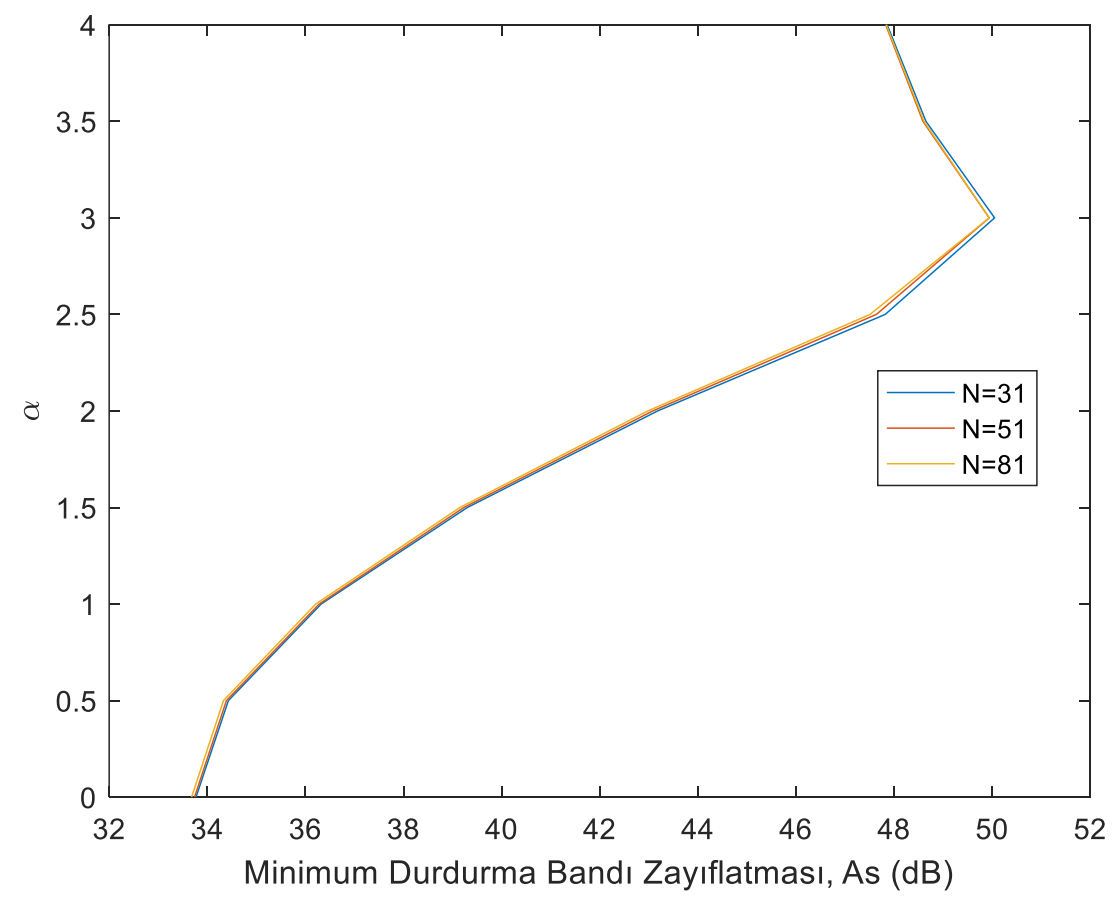

Şekil 7. Farklı süzgeç uzunlukları için önerilen pencereyle tasarlanmış süzgeçlerde $\alpha$ ve $A_{s}$ ilişkisi

Önerilen pencere ile tasarlanmış süzgeçlerde, önerilen pencere parametreleri ile süzgeç parametreleri arasında incelenecek ikinci ilişki $\mathrm{N}, \Delta \omega$ ve $\mathrm{A}_{\mathrm{s}}$ arasındaki ilişkidir. Bu ilişkiyi iki parametreye indirmek için $\mathrm{N}$ ve $\Delta \omega$ cinsinden tanımlanmış ve aşağıdaki eşitlikle verilen normalize süzgeç geçiş bant-genişliği parametresi kullanılmaktadır [7].

$$
D_{f}=\Delta \omega(N-1) / 2 \pi
$$

Dolayısıyla, incelenecek ikinci ilişki $\mathrm{D}_{\mathrm{f}}$ ve $\mathrm{A}_{\mathrm{s}}$ arasındaki ilişkidir. Bunun için farklı süzgeç uzunlukları için birçok sayısal süzgeç örneğinden elde edilmiş $\mathrm{D}_{\mathrm{f}}$ ve $\mathrm{A}_{\mathrm{s}}$ arasındaki ilişki Şekil 8'de verilmiştir. Bu şekilde 
görüleceği üzere yaklaşı olarak $\mathrm{D}_{\mathrm{f}}>2.83$ için $\mathrm{A}_{\mathrm{s}}$ değerinin artık artmadığı ve $\mathrm{A}_{\mathrm{s}}$ değerinin $50 \mathrm{~dB}$ 'i geçmediği görülmektedir.

$\mathrm{Bu}$ kısımda yapılan analizlerden elde edilen sonuç olarak, önerilen Kaiser-Hann penceresinin $\alpha \leq 3$, $\mathrm{A}_{\mathrm{s}} \leq 50 \mathrm{~dB}$ ve $\mathrm{D}_{\mathrm{f}} \leq 2.83$ aralıklarında daha kullanışlı bir süzgeçleme performansı sergilediği görülmüştür.

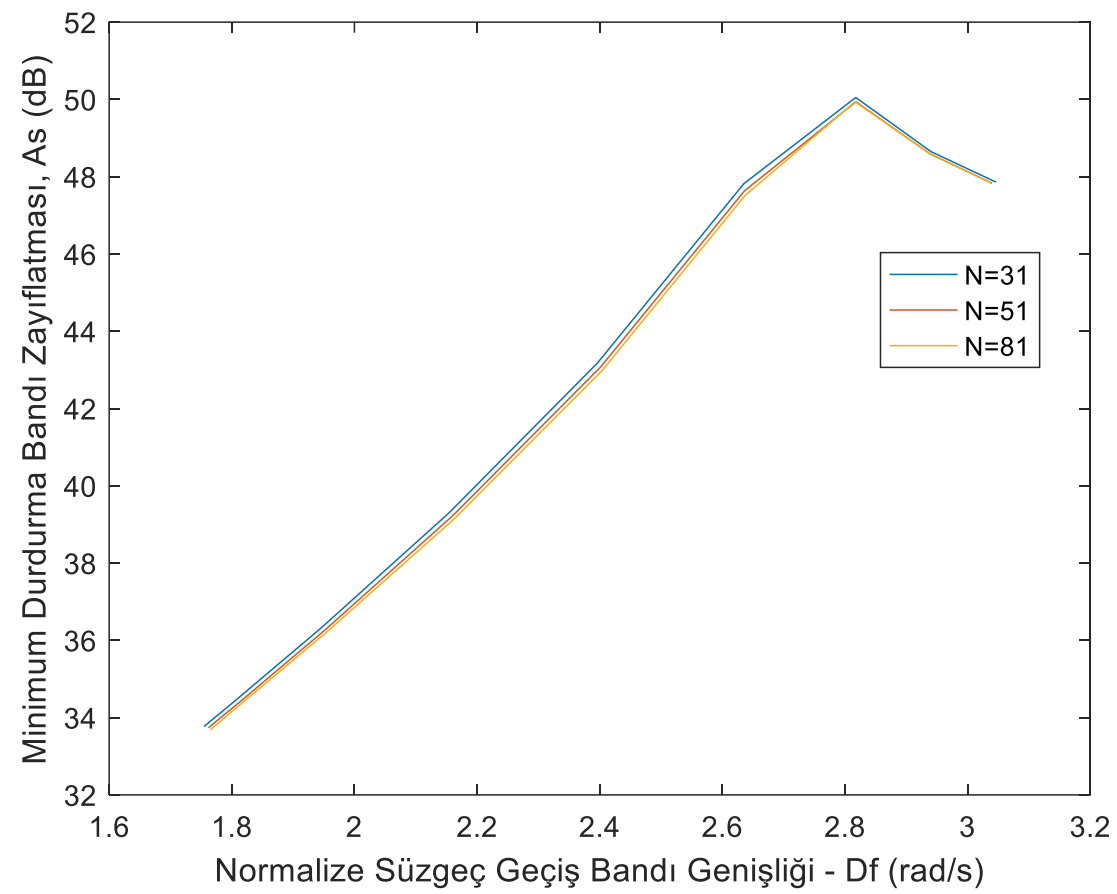

Şekil 8. Farkl süzgeç uzunluklart için önerilen pencereyle tasarlanan süzgeçlerde $A_{s}$ ve $D_{f}$ ilişkisi

\subsection{Karşılaştırmalı Süzgeç Tasarım Örnekleri (Comparative Filter Design Examples)}

$\mathrm{Bu}$ kısımda, önerilen pencere ile tasarlanan sayısal süzgeçlerin performansını değerlendirmek için literatürdeki diğer iki parametreli pencereler olan Kaiser-hamming, Cosh-Hamming, ExponentialHamming, Saramaki, Kaiser, Dolph-Chebyshev, Cosh, Exponential, Gaussian, Hann-Poisson ve Poisson pencereleriyle tasarlanan süzgeçlerle karşılaştırmaları verilmektedir.

İlk karşılaştırma olarak önerilen pencere ile tasarlanan süzgeçler Kaiser penceresiyle tasarlanan süzgeçlerle karşılaştırılmıştır. Şekil 9, Şekil 10 ve Şekil 11'de sırasıyla 31, 51 ve 81 süzgeç uzunlukları için birçok süzgeç örneğinden elde edilmiş $\mathrm{D}_{\mathrm{f}}$ ve $\mathrm{A}_{\mathrm{s}}$ arasındaki ilişkiler verilmiştir. Şekillerden görüleceği üzere $\mathrm{D}_{\mathrm{f}} \leq 2.90$ aralığı için önerilen pencereyle tasarlanan süzgeçler Kaiser penceresine göre daha iyi sonuçlar vermektedir.

İkinci karşılaştırma olarak önerilen pencere ile tasarlanan süzgeç ile literatürdeki diğer iki parametreli pencereler olan Kaiser-Hamming, Cosh-Hamming, Exponential-Hamming, Saramaki, Kaiser, DolphChebychev, Cosh, Exponential, Gaussian, Hann-Poisson ve Poisson pencereleriyle tasarlanan süzgeçler karşılaştırılmıştır. $\mathrm{N}=51$ süzgeç uzunluğu için $\Delta \omega=0,346 \mathrm{rad} / \mathrm{s}(\approx 0,11 \pi)$ geçiş bant-genişliğine ve $\omega_{c}=0,4 \pi \mathrm{rad} / \mathrm{s}$ kesme frekansına sahip farklı pencerelerle tasarlanmış alçak geçiren süzgeçlerin genlik spektrumları Şekil 12, Şekil 13 ve Şekil 14'te verilmiştir. Bu şekillerden elde edilen ilgili süzgeç spektral değerleri Tablo 2'de ayrıca sunulmuş̧tur. Görüldüğü gibi önerilen pencere, verilen örnek tasarım için en iyi $\mathrm{A}_{\mathrm{s}}$ değerini sağlamaktadır. 


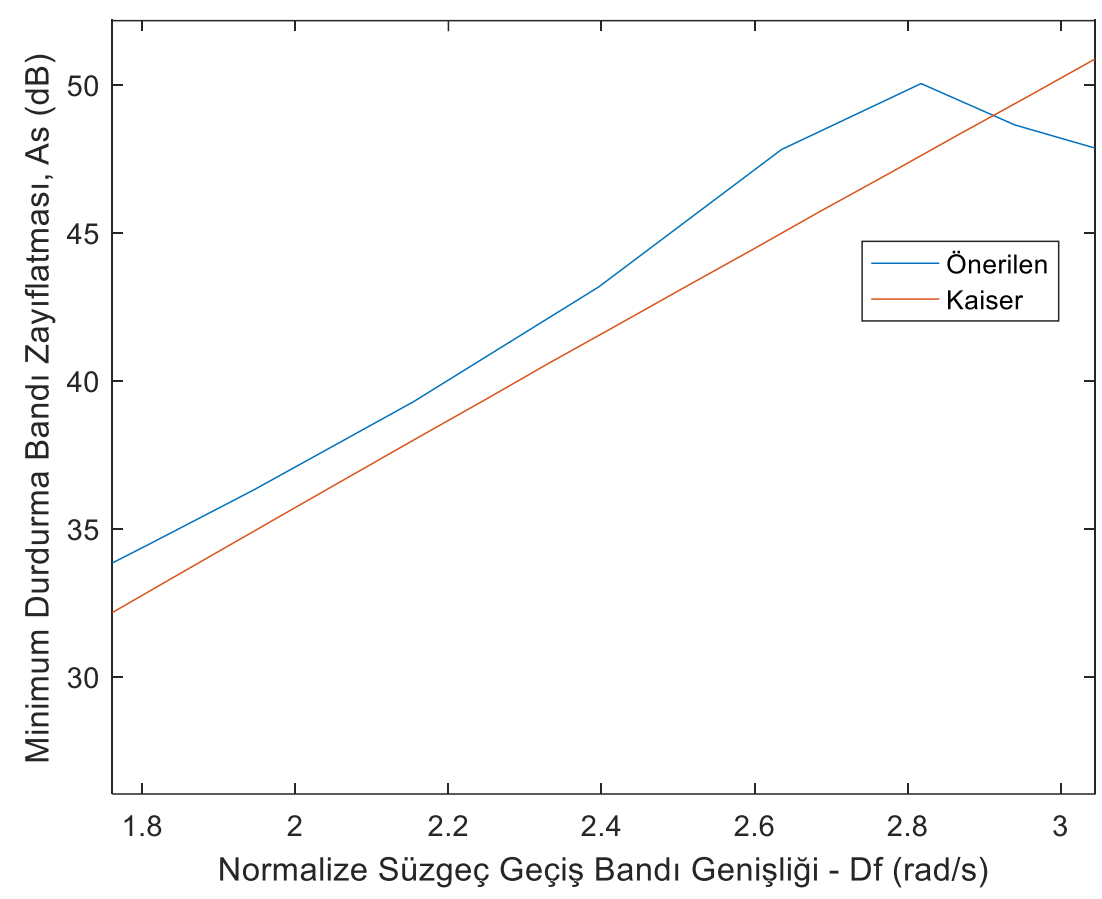

Şekil 9. $N=31$ için önerilen ve Kaiser pencereleriyle tasarlanan süzgeçlerin $D_{f}-A_{s}$ karakteristikleri karşılaştırılması

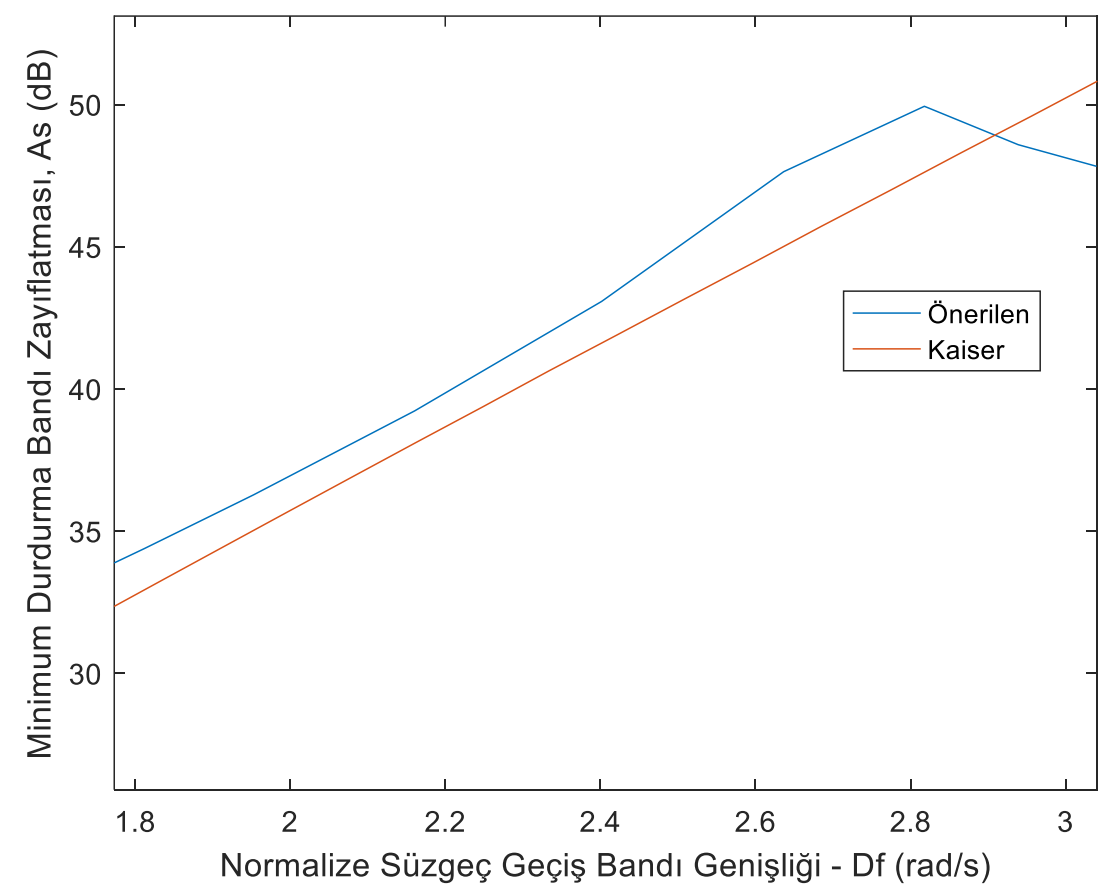

Şekil 10. $N=51$ için önerilen ve Kaiser pencereleriyle tasarlanan süzgeçlerin $D_{f}-A_{s}$ karakteristikleri karşılaş̧ırılması 


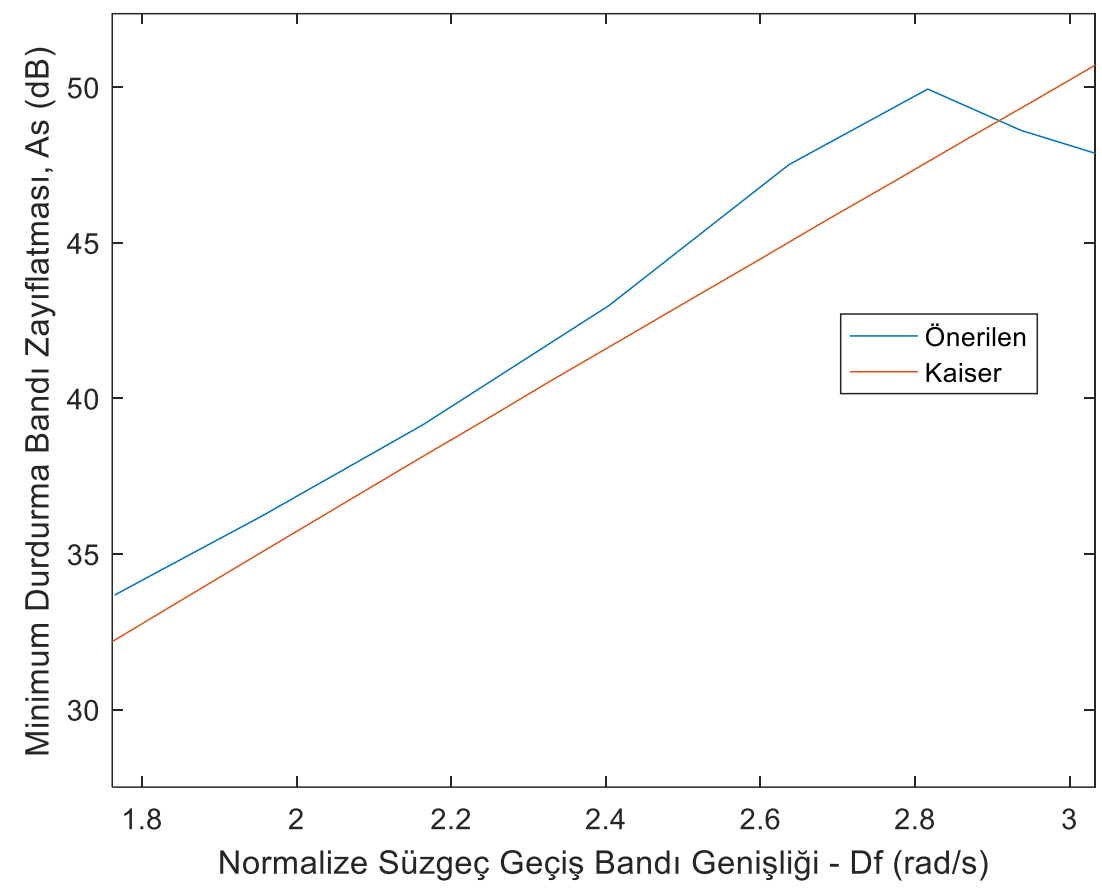

Şekil 11. $N=81$ için önerilen ve Kaiser pencereleriyle tasarlanan süzgeçlerin $D_{f^{-}} A_{s}$ karakteristikleri karşılaştırılması

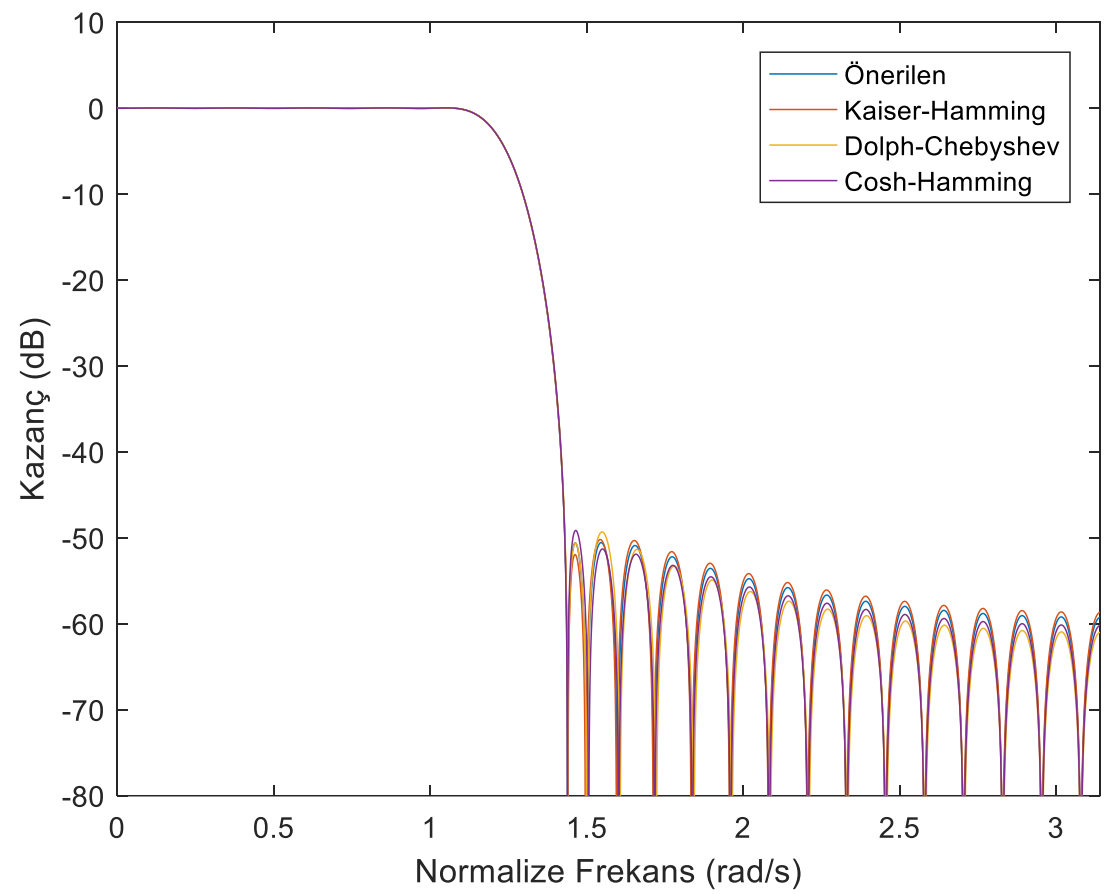

Şekil 12. $N=51$ için önerilen, Kaiser-Hamming, Dolph-Cheyshev ve Cosh-Hamming pencereleriyle oluşturulan süzgeçler 


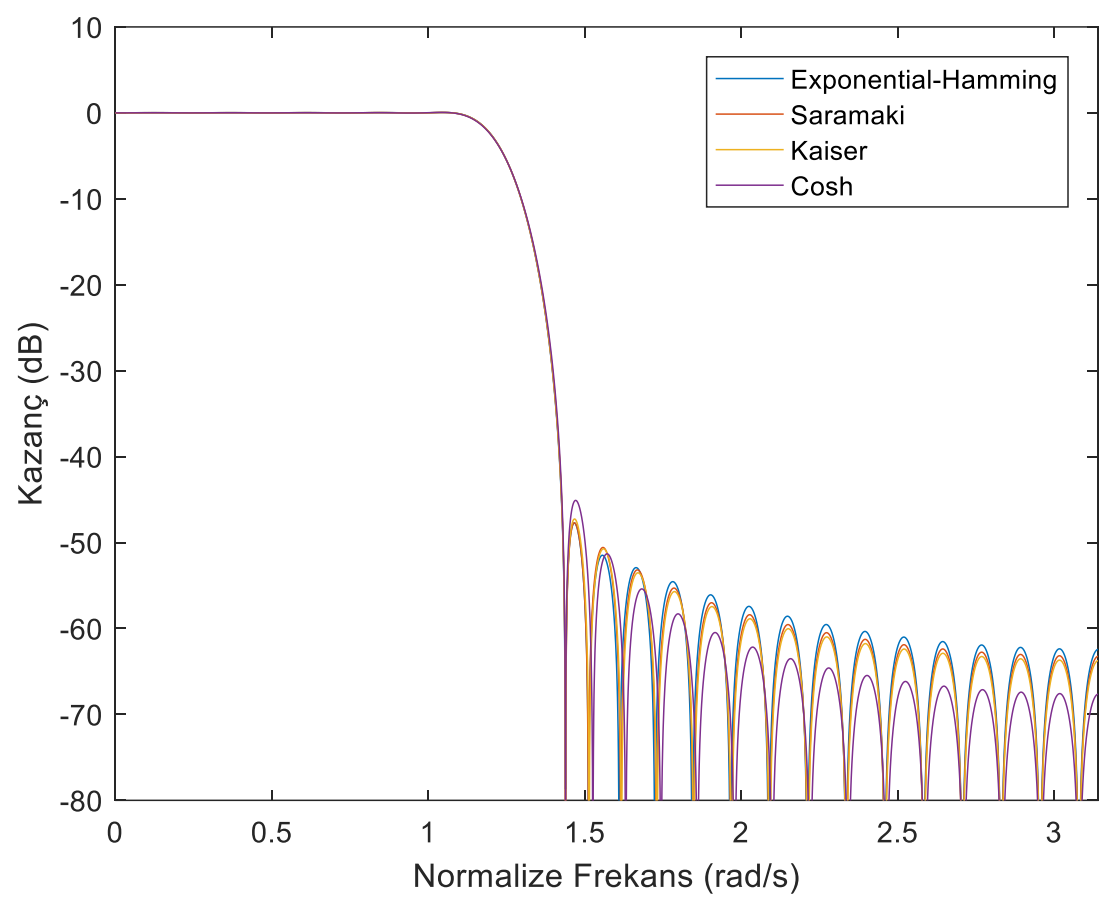

Şekil 13. $N=51$ için Exponential-Hamming, Saramaki, Kaiser ve Cosh pencereleriyle oluşturulan süzgeçler

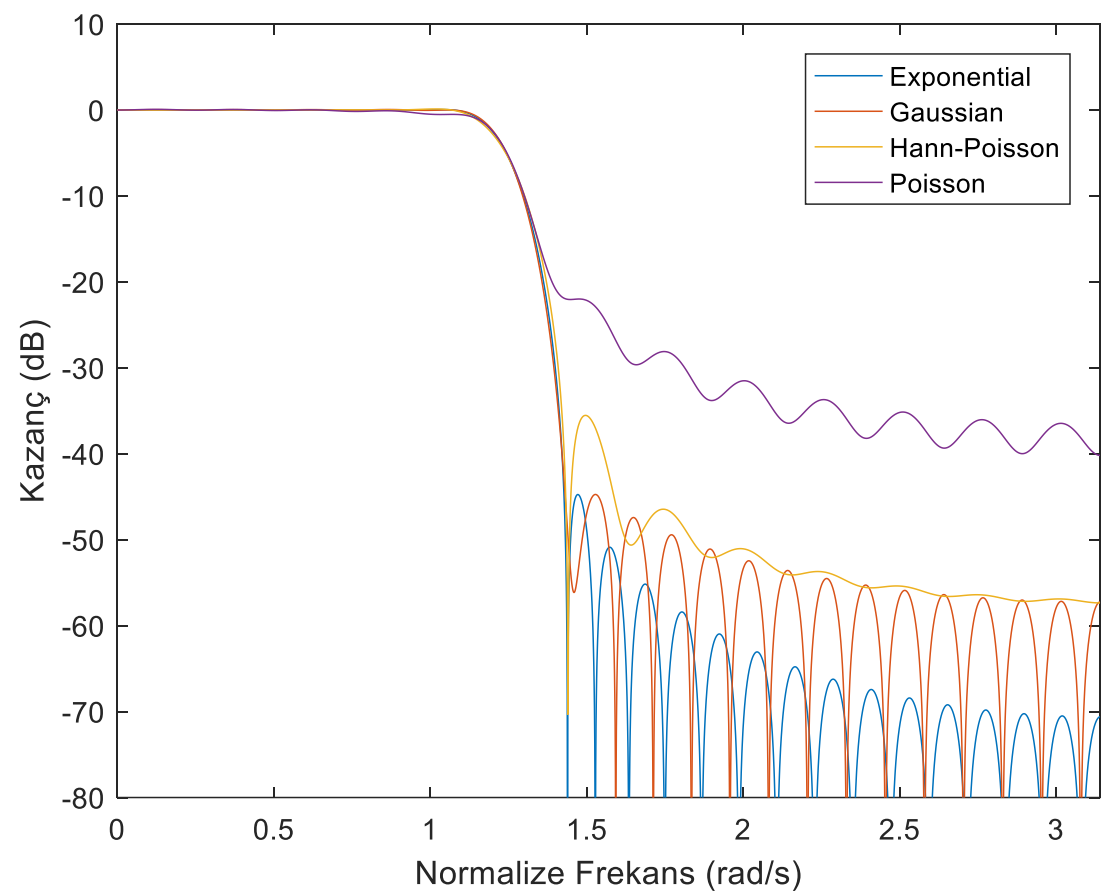

Şekil 14. $N=51$ için Exponential, Gaussian, Hann-Poisson ve Poisson pencereleriyle oluşturulan süzgeçler 
Tablo 2. Önerilen pencere ve literatürdeki iki parametreli pencereler ile tasarlanan süzgeçlerin spektral değerlerinin karşılaştırılması

\begin{tabular}{|l|c|c|c|c|}
\hline Pencere & $\mathbf{N}$ & $\boldsymbol{\Delta \omega}$ & $\boldsymbol{\omega}_{\boldsymbol{c}}$ & $\mathbf{A}_{\mathbf{s}}$ \\
\hline Önerilen & 51 & $0,11 \pi$ & $0,4 \pi$ & 50,54 \\
\hline Kaiser-Hamming [13] & 51 & $0,11 \pi$ & $0,4 \pi$ & 50,19 \\
\hline Dolph-Chebyshev [2] & 51 & $0,11 \pi$ & $0,4 \pi$ & 49,30 \\
\hline Cosh-Hamming [14] & 51 & $0,11 \pi$ & $0,4 \pi$ & 49,13 \\
\hline Exponential-Hamming [15] & 51 & $0,11 \pi$ & $0,4 \pi$ & 47,74 \\
\hline Saramaki [6] & 51 & $0,11 \pi$ & $0,4 \pi$ & 47,70 \\
\hline Kaiser [4] & 51 & $0,11 \pi$ & $0,4 \pi$ & 47,26 \\
\hline Cosh [9] & 51 & $0,11 \pi$ & $0,4 \pi$ & 45,10 \\
\hline Exponential [11] & 51 & $0,11 \pi$ & $0,4 \pi$ & 44,74 \\
\hline Gaussian [5] & 51 & $0,11 \pi$ & $0,4 \pi$ & 44,73 \\
\hline Hann-Poisson [5] & 51 & $0,11 \pi$ & $0,4 \pi$ & 35,53 \\
\hline Poisson [3,5] & 51 & $0,11 \pi$ & $0,4 \pi$ & 21,99 \\
\hline
\end{tabular}

\section{SONUÇ (CONCLUSION)}

$\mathrm{Bu}$ çalışmada, Kaiser ve Von-Hann pencerelerinden türetilen yeni bir hibrit pencere önerilmiş ve kısaca Kaiser-Hann penceresi olarak adlandırılmış bu pencerenin sayısal süzgeç uygulamalarındaki performansı kıyaslamalı olarak analiz edilmiştir. Önerilen pencerenin $N$ ve $\alpha$ gibi iki bağımsız parametreye sahip olması nedeniyle, sabit bir $\mathrm{N}$ değeri için sadece $\alpha$ değerinin değiştirilmesiyle sabit bir süzgeç uzunluğunda birçok farklı karakteristikte süzgeç tasarımı elde edilebilir.

Performans analizi için yapılan benzeşim sonuçları, önerilen pencerenin $A_{s} \leq 50 \mathrm{~dB}$ için $\alpha \leq 3$ ve $\mathrm{D}_{\mathrm{f}} \leq 2.83$ aralıklarında en iyi sonuçları verdiğini göstermiştir. Farklı süzgeç uzunlukları için Kaiser penceresi ile yapılan karşılaştırma örneklerinde ise $\mathrm{D}_{\mathrm{f}} \leq 2.90$ aralığ için önerilen pencereyle tasarlanan süzgeçler Kaiser penceresi ile tasarlanan süzgeçlere göre daha iyi $\mathrm{A}_{s}$ değeri vermiş, dolayısıyla önerilen pencereler $\mathrm{D}_{\mathrm{f}} \leq 2.90$ aralığ 1 için Kaiser penceresine tercih edilebileceği sonucuna varılmıştır. İkinci karşılaştırma örneği olarak, önerilen pencere ile tasarlanan süzgeç literatürdeki diğer iki parametreli pencereler olan Kaiser-Hamming, Cosh-Hamming, Exponential-Hamming, Saramaki, Kaiser, Dolph-Chebychev, Cosh, Exponential, Gaussian, Hann-Poisson ve Poisson pencereleriyle tasarlanan süzgeçler ile karşılaştırılmıştır. Sonuç olarak, sabit bir süzgeç uzunluğu ve geçiş bant-genişliğinde en iyi minimum durdurma-bandı zayıflatmasını (dolayısıyla en iyi tasarımı) önerilen pencere ile tasarlanan sayısal süzgeç vermiştir.

Önerilen pencere ile tasarlanan sayısal süzgeçler belirli bir süzgeç spektrum parametresi aralığında Kaiser penceresinden daha iyi sonuçlar verdiğinden, önerilen pencere Kaiser penceresi yerine haberleşme, biyomedikal ve imge işleme [16-22] gibi alanlarda kullanılabilir.

\section{KAYNAKLAR (REFERENCES)}

[1] A. Antoniou, Digital Signal Processing: Signal, Systems, and Filters, McGraw-Hill, USA, 2005.

[2] C. L. Dolph, A Current Distribution for Broadside Arrays Which Optimizes the Relationship Between Beamwidth and Side-Lobe Level, Proc. IRE, 34:335-348, 1946.

[3] N. K. Bary. A Treatise on Trigonometric Series, Vol.I, MacMillan, New York, 1964.

[4] J. F. Kaiser, Nonrecursive Digital Filter Design Using I0-Sinh Window Function, Proceedings of IEEE Int Symp Circuits and Systems (ISCAS'74), San Francisco, Calif, USA, 20-23, 1974. 
[5] F. J. Harris, On the Use of Windows for Harmonic Analysis with the Discrete Fourier Transform, Proc. IEEE, 66:51-83, 1978.

[6] T. Saramaki, A Class of Window Functions with Nearly Minimum Sidelobe Energy for Designing FIR Filters, Proc. IEEE Int. Symp. Circuits and Systems, Portland, Ore, USA, 359-362, 1989.

[7] S. W. A. Bergen, A. Antoniou, Design of Nonrecursive Digital Filters Using the Ultraspherical Window Function, EURASIP Journal on Appl. Signal Proc., 12:1910-1922, 2005.

[8] K. Avci, A. Nacaroğlu, High Quality Low Order Nonrecursive Digital Filter Design Using Modified Kaiser Window, Proc. of CSNDSP'08, Graz, Austria, 239-242, 23-25 Temmuz 2008.

[9] K. Avci, A. Nacaroğlu, Cosh Window Family and its Application to FIR Filter Design, Int. Journal of Electronics and Communications, 63(11):907-916, 2009

[10] M. Mottaghi-Kashtiban, M. G. Shayesteh, New Efficient Window Function, Replacement for the Hamming Window, IET Signal Processing, 5(5):499-505, 2011.

[11] K. Avci, A. Nacaroğlu, Design of Nonrecursive Digital Filters Using the Exponential Window, Int. J. of Advanced Electrical \& Electronics Engineering, 2(2):308-316, 2013.

[12] R. Pachauri, R. Saxena, S. N. Sharma, Studies on Z-Window Based FIR Filters, ISRN Signal Processing, 1-8, 2013.

[13] K. Avci, Kaiser-Hamming Window and its Performance Analysis for Nonrecursive Digital Filter Design, Journal of the Faculty of Engineering and Architecture of Gazi University, 29(4):823-833, 2014.

[14] B. Üstün, K. Avci, Yinelemesiz Sayısal Süzgeç Tasarımı için Cosh ve Hamming Pencereleri Tabanlı Yeni bir Hibrit Pencere, 23. IEEE Sinyal İşleme ve İletişim Uygulamaları Kurultayı (SIU 2015), Malatya, 2282-2285, 16-19 May1s, 2015.

[15] K. Avci, Design of FIR Filters Using Exponential-Hamming Window Family, Turkish J Elec Eng \& Comp Sci, 24(4):2513-2524, 2016.

[16] K. M. M. Prabhu, Window Functions and Their Applications in Signal Proccesing, CRC Press, Boca Raton, USA, 2014.

[17] K. Avci, E. Gümüşsoy, Üstel Penceresi Tabanlı M-Kanallı Kosinüs Modüleli Süzgeç Öbeklerinin Tasarımı, 24. IEEE Sinyal İşleme ve İletişim Uygulamaları Kurultayı (SIUU 2016), Zonguldak, 845-848, 16-19 May1s, 2016.

[18] S. R. Seydnejad, R. I. Kitney, Real-Time Heart Rate Variability Extraction Using the Kaiser Window, IEEE T Bio-Med Eng, 44(10):990-1005, 1997.

[19] D. Ashutosh, J. Alok, C. S. Pramod, Design and Performance Analysis of Adjustable Window Functions Based Cosine Modulated Filter Banks, Digit Signal Process, 23(1):412-417, 2013.

[20] S. Ramkumar, J. Alok, S. Rajiv, An Optimized Design of Nonuniform Filter Bank Using VariableCombinational Window Function, AEU-Int J Electron C, 67(7):595-601, 2013.

[21] S. Aljahdali, M. Nofal, Y. Albagory, A modified Array Processing Technique Based on Kaiser Window for Concentric Circular Arrays, International Conference on Multimedia Computing and Systems (ICMCS), Tangier, Fas, 458-461, 10-12 May1s 2012.

[22] L. Sumamo, Handwritten Word Segmentation Using Kaiser Window, International Conference on Quality in Research (QIR), Yogyakarta, Endonezya, 73-78, 25-28 Haziran 2013. 